Gut, 1971, 12, 369-371

\title{
Observations on iron in the jejunal lumen after a standard meal
}

\author{
J. GLOVER AND A. JACOBS
}

From the Department of Haematology, Welsh National School of Medicine, Cardiff

SUMMARY For two hours after the ingestion of a standard meal with an iron content of $3.45 \mathrm{mg}$ there is a rise in iron concentration in the jejunal lumen. Initially this is in a high molecular weight form but there is a steady increase in the concentration of low molecular weight compounds. This is consistent with the gradual intraluminal transfer of iron from a mucopolysaccharide carrier to ${ }^{-}$low molecular weight ligands before absorption.

Under normal conditions much of the iron present in ingested food is released as a result of peptic digestion in the stomach. This iron is largely in an ionisable form except after the digestion of meat when a significant amount of haem iron is liberated (Jacobs and Greenman, 1969). It has been suggested that at the acid $p \mathrm{H}$ of the stomach the ionisable iron exists in solution in a chemically reactive form. When the gastric contents are neutralized most of the iron present combines with gastric mucopolysaccharide to form a soluble high molecular weight complex (Jacobs and Miles, 1969). This prevents precipitation of iron in the small intestine and maintains it in a form available for uptake by the mucosa. It is not clear how iron passes from the mucopolysaccharide carrier into the epithelial cells of the intestinal villi. Fractionation of the epithelial cells during the early phase of iron absorption in the rat shows no evidence of iron uptake by pinocytosis (Worwood and Jacobs, 1971) and it seems likely that the iron bound to the gastric carrier in the lumen is detached in some way before entering the epithelial cells. Studies in vitro have shown that iron may be detached from its intraluminal carrier by a number of small molecules of which cysteine and ascorbic acid are the most active (Jacobs and Miles, 1969). Other sugars and amino acids may act as iron chelators when the molar ratio of ligands to iron is high enough. The present study was carried out in order to determine the concentration of iron present in solution in the jejunal contents after a standard meal with a known iron content and whether the iron liberated was still attached to a mucopoly-

Received for publication 16 March 1971. saccharide carrier or whether it assumed a low molecular weight form.

\section{Methods}

Ten healthy adults, who had no history of gastrointestinal disease, were studied. Their fully informed consent was obtained in each case.

After an overnight fast, a Portex polyvinyl tube with an external diameter of $5 \mathrm{~mm}$ was passed into the upper jejunum and its position was checked radiologically. All samples were taken with the tip of the tube in the first 12 inches of the jejunum. Jejunal contents were aspirated for $30 \mathrm{~min}$ to obtain a basal sample and at the end of that time the subject was given a standard meal consisting of shepherd's pie, peas, and a drink of water. This meal had the same composition as the one used previously (Jacobs and and Miles, 1969) and had a total iron content of $3.45 \mathrm{mg}$. Sampling continued for a further two hours.

Total iron in the samples was estimated by the method of Tennant and Greenman (1969). Haem iron was estimated by the method of Crosby and Furth (1956). Ionisable iron was estimated by a modification of the method of Young and Hicks (1965).

An aliquot of each sample of jejunal contents was dialysed for 18 hours against $\mathbf{0} \cdot 15$ molar barbitoneacetate buffer at the same $p H$ as the samples. Visking tubing grade 18DC was used which is permeable to substances of molecular weight less than 10,000 . In a small number of cases the supernatant obtained after centrifugation of jejunal contents was subjected to ultrafiltration through membranes of known pore size. Some of the results 
were confirmed by gel filtration using Sephadex G25.

\section{Results}

Changes in jejunal $p \mathrm{H}$ and iron concentration are shown in Table $\mathrm{I}$. The mean $p \mathrm{H}$ of basal samples of jejunal juice was 6.8 and this decreased slightly during the two hours following administration of the test meal. The mean total iron content of basal jejunal juice was $47 \mu \mathrm{g} / 100 \mathrm{ml}$ and this rose following administration of the test meal. Mean haem iron concentrations were low throughout, the maximum being $21 \mu \mathrm{g} / 100 \mathrm{ml}$, occurring half an hour after ingestion of the test meal.

\begin{tabular}{llcl}
\hline Time after Meal $(\mathrm{hr})$ & $\mathrm{pH}$ & $\begin{array}{l}\text { Total Iron } \\
(\mu \mathrm{g} / 100 \mathrm{ml})\end{array}$ & $\begin{array}{l}\text { Haem iron } \\
(\mu \mathrm{g} / 100 \mathrm{ml})\end{array}$ \\
\hline Basal & $6.8 \pm 0.2$ & $47 \pm 9$ & $12 \pm 4$ \\
$\frac{1}{1}$ & $6.1 \pm 0.2$ & $122 \pm 32$ & $21 \pm 9$ \\
1 & $5.9 \pm 0.2$ & $109 \pm 25$ & $13 \pm 5$ \\
$1 \frac{1}{2}$ & $6.0 \pm 0.3$ & $105 \pm 17$ & $17 \pm 8$ \\
2 & $5.5 \pm 0.3$ & $6 \pm 3$ \\
\hline
\end{tabular}

Table Mean $\mathrm{pH}$ and total iron concentration in jejunal contents after a standard test meal

Measurement of the amount of iron in each sample which remained after dialysis allowed the total ionisable iron concentration in each sample to be differentiated into high and low molecular weight compounds. The mean concentration of each of these forms after administration of a meal is shown in Figure 1. Immediately after the meal there is a rise in the amount of large molecular weight iron in the jejunal contents but as time progresses there is a gradual increase in the concentration of low molecular weight iron compounds.

Some of the jejunal samples were filtered through a Sartorius membrane allowing the passage of substances with a molecular weight of 10,000 . When the filtrate was applied to a Sephadex G25 column equilibrated with buffer at the same $p \mathrm{H}$ as the sample all the iron was eluted in the bed volume indicating a molecular weight of less than 2,000. Similarly, passage of such a filtrate through an Amicon membrane (UM 05) designed to hold back compounds of a molecular weight over 500 resulted in the passage of a considerable proportion of the iron present.

\section{Discussion}

Under fasting condition there is not a great deal of iron present in the upper jejunum. That which is present is in the form of high molecular weight compounds and much of it is probably endogenous in origin. After the standard meal the iron content of the jejunum rises and initially most of this iron is in a high molecular weight form. During the two hours after the meal a low molecular weight form of iron becomes detectable in the lumen and increases until two hours after the meal when it comprises $50 \%$ of the ionisable iron present.

It is known that the iron compound formed when the products of peptic digestion are neutraliezd is a high molecular weight mucopolysaccharide complex (Jacobs and Miles, 1969). It is not known how iron

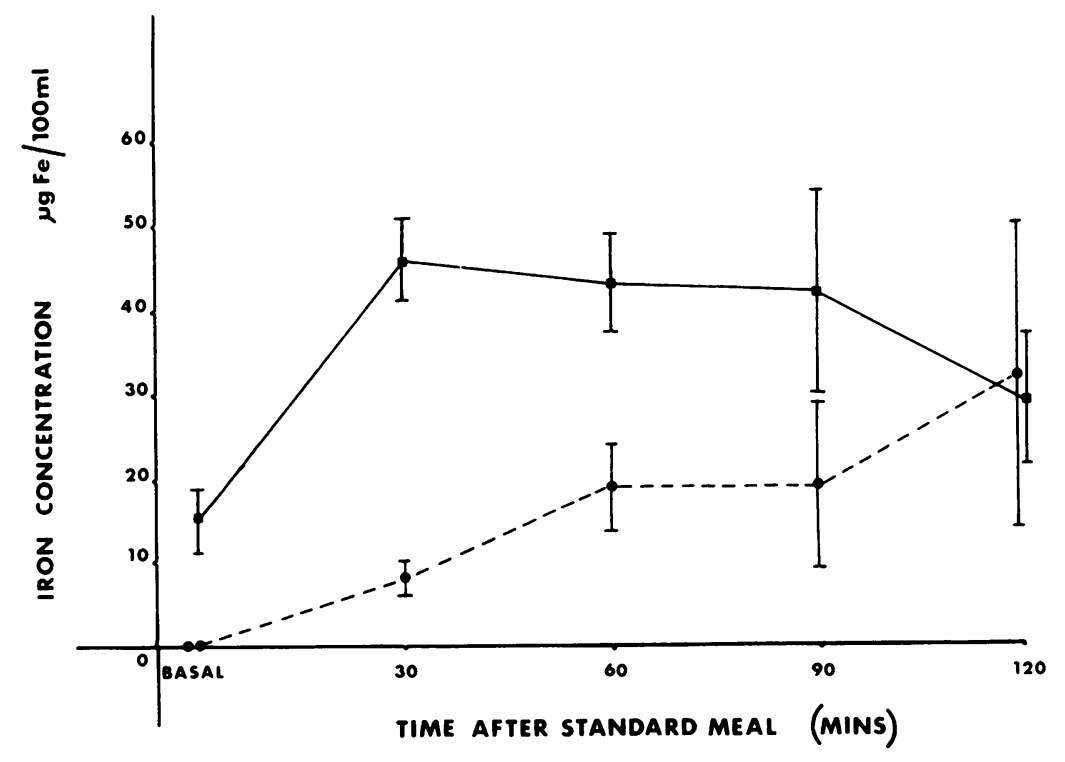

Fig. Mean concentrations $( \pm S D)$ of ionisable iron in the jejunal lumen following a standard meal.

$\square=$ High molecular weight compounds; wew molecular weight compounds. 
is released from this mucopolysaccharide carrier which presumably passes into the jejunum and becomes available to the intestinal cells. There are a number of possible sources for the sugars, amino acids, and dipeptides which could chelate iron and remove it from its carrier. They may be produced in the lumen of the jejunum as the result of pancreatic digestion of food substances and in this case the transfer of iron from a high molecular form to a low molecular form would be expected to occur in the lumen of the gut. Sugars and amino acids may also be formed in the brush border as part of the digestive process and in these circumstances the high local concentration between the microvilli would give optimal conditions for chelation and absorption in association with compounds which might not necessarily chelate iron in the lumen. A third source of potential iron chelators is from the epithelial cells themselves which are known to secrete amino acids into the lumen, and presumably these also will be present in a relatively high concentration in the brush border.

The present results suggest that immediately after a meal the iron in the jejunal lumen is largely in a high molecular weight form and the gradual increase in low molecular weight iron suggests that the transfer from the mucopolysaccharide carrier is taking place within the lumen of the gut. This does not of course rule out the possiblity that chelation may also occur at the brush border. The nature of the ligands involved in the removal of iron from its carrier is unknown but studies in vitro have shown that cysteine is highly effective, and it is of interest that Martinez-Torres and Layrisse (1970) have shown that the stimulating effect of fish on iron absorption appears to be due to the presence of cysteine in the digestive breakdown products.

It now appears justified to consider that the chemical form of iron being presented to the epithelial cells of the small intestine is at least partly a low molecular weight iron complex, and consideration of intraepithelial mechanisms of absorption should take this into account.

\section{References}

Crosby, W. H., and Furth, F. W. (1956). A modification of the benzidine method for measurement of hemoglobin in plasma and urine. Blood, 11, 380-383.

Jacobs, A., and Greenman, D. A. (1969). Availability of food iron. Brit. med.J., 1, 673-676.

Jacobs, A., and Miles, P. M. (1969). Intraluminal transport of iron from stomach to small intestinal mucosa. Brit. med. J., 4, 778-781.

Martinez-Torres, C., and Layrisse, M. (1970). Effect of amino-acids on iron absorption from a stable vegetable food. Blood, 35, 669-682.

Tennant, G. B., and Greenman, D. A. (1969). Determination of iron solutions containing iron complexes. J. clin. Path., 22, 301-303.

Worwood, M., and Jacobs, A. (1971). The subcellular distribution of ${ }^{50} \mathrm{Fe}$ during iron absorption in the rat. Brit. J. Haemat., 20, 587-597.

Young, D. S., and Hicks, J. M. (1965). Method for the automatic determination of serum iron. J. clin. Path., 18, 98-102. 